\title{
Long-term Earth Orientation Monitoring Using Various Techniques
}

\author{
D. Gambis \\ IERS/CB and UMR8630, Paris Observatory, Paris, France
}

\begin{abstract}
A continuous composite series of polar motion components extending from 1846 until now called EOP (IERS) C01 is available at the Earth Orientation Section of the Central Bureau of the IERS. This series is the basis of the IERS system. It relies on different series derived from optical astrometry until 1972 and geodetic techniques since. It is given at 0.1 year intervals (1846-1889) and 0.05 year intervals (1890-now). Its accuracy has dramatically improved from 100 mas in 1846 to about 0.2 mas at present.

Now the IERS combined solutions involve mainly the contributions of VLBI, GPS and SLR techniques. It is regularly recomputed to take advantage of the improvement of the various recent individual contributions and of the refinement of the analyses procedures.

The objective of this paper is to describe this long-term polar motion series and to present the evolution and the state of the art of the multi-technique EOP combined solutions and the predictions regularly computed at the IERS/CB.
\end{abstract}

\section{Introduction}

Euler, in 1765, considering the Earth as a rigid body theorized that the pole would rotate with respect to the crust in 305 days. However for more than one century, astronomers were not able to detect this variation in their observations. At the end of the last century, international programs of observation of the polar motion variations were initiated by astronomers and geodesists, with at first the International Latitude Service (ILS, 1899) based on the observations of 5 or 6 zenith telescopes. In 1962 the International Polar Motion Service, IPMS was created. It had the task to monitor polar motion using the observations of all available optical instruments. In 1912, the Bureau International de l'Heure (BIH) was created. In 1955, it was charged to run the rapid service (Service International Rapide des Latitudes, SIR) to derive a rapid polar motion solution. The International Earth Rotation Service (IERS) created by the International Union of Geodesy and Geophysics (IUGG) and the International Astronomical Union (IAU) began operation in 1988. It is in charge of providing basic references to the international scientific community linked mainly to astronomy, geodesy, space sciences and geophysics. It is being reorganized in 2000 , for a better synergy between the different components in particular with the new techniquebased Services (IGS, IVS and ILRS). 


\section{Long-term Polar Motion solution}

The contributions of the observing techniques to the Earth Rotation monitoring and the evolution of the accuracies obtained since 1846 are summarized in Table 1. For many decades, the observations were made using mostly visual and photographic zenith telescopes. Since the advent of the space era in the $1960 \mathrm{~s}$, new geodetic techniques were applied for geodynamics. Now, the global observing activity involves Very Long Baseline Radio Interferometry (VLBI), Lunar and Satellite Laser Ranging (LLR, SLR), Global Positioning System (GPS) and more recently Doppler Orbit Determination and Radiopositioning Integrated on Satellite (DORIS).

A continuous series of Polar Motion components extending from 1846 until now is available at the Central Bureau of the IERS (EOP(IERS) C01). This series is based on the following solutions:

1846-1899 : Fedorov et al. (1972) solution derived from three series of absolute declination programs (Pulkovo, Greenwhich, Washington).

1900-1961: Vondrák et al. (1995) solution derived from optical astrometry analyses based on the Hipparcos reference frame.

1962-now : IERS and BIH solutions. Space techniques were introduced in 1969 for Universal Time (Lunar Laser Ranging) and in 1972 for polar motion (Satellite Doppler Tracking).

The main characteristics of the various contributions (time resolution, precision) are given in the following table.

Table 1. Evolution of the contribution of the various techniques to the Earth orientation parameters determination. PM : polar motion $(\mathrm{x}, \mathrm{y}), \mathrm{UT}$ : universal time UT1, CP : celestial pole coordinates. The numbers of * roughly reflect the level of contribution (precision and density of measurements).

\begin{tabular}{|c|c|c|c|c|c|c|c|c|c|}
\hline Period & & Opt.Astr. & LLR & Doppler & $\overline{\text { SLR }}$ & VLBI & GPS & resol. & Precision \\
\hline $1846-1899$ & PM & * & & & & & & month & 0.10 \\
\hline $1900-1961$ & PM & * & & & & & & month & 0.05 \\
\hline \multirow{3}{*}{$1962-1971$} & PM & \#* & & & & & & \multirow{3}{*}{ weeks } & $0 ! 02$ \\
\hline & & & & & & & & & \\
\hline & UT & ** & & & & & & & $0.002 \mathrm{~s}$ \\
\hline \multirow{2}{*}{$1972-1979$} & $\mathrm{PM}$ & ** & & ** & & & & \multirow{2}{*}{ week } & $0 . ! 01$ \\
\hline & UT & ** & ** & & & & & & $0.001 \mathrm{~s}$ \\
\hline \multirow{3}{*}{$1980-1983$} & PM & & & & ****; & & & \multirow{3}{*}{ week } & $0 !^{\prime \prime} 002$ \\
\hline & & & & & & & & & \\
\hline & UT & & * & & * & ** & & & $0.0004 \mathrm{~s}$ \\
\hline \multirow{3}{*}{$1984-1994$} & PM & & & & \$* & ** & & \multirow{3}{*}{ days } & 0,0005 \\
\hline & $\mathrm{CP}$ & & & & & $* * * *$ & & & 0."0005 \\
\hline & UT & & & & $*$ & $* * *$ & & & $0.00005 \mathrm{~s}$ \\
\hline \multirow{3}{*}{ 1995-1999 } & PM & & & & \$** & **** & *** & \multirow{3}{*}{ day } & 0.0003 \\
\hline & $\mathrm{CP}$ & & & & & $* * * *$ & & & $0 .^{\prime \prime} 0003$ \\
\hline & UT & & & & * & $* * *$ & * & & $0.00003 \mathrm{~s}$ \\
\hline
\end{tabular}

Figure 1 shows the $\mathrm{x}$ and $\mathrm{y}$ pole components since 1846. The continuous line shows the so-called and still controversial "secular polar motion" described and discussed in various papers (Vondrák et al., 1995). The periodic part is 
Polar motion: CO1 series, UNIT = 1"

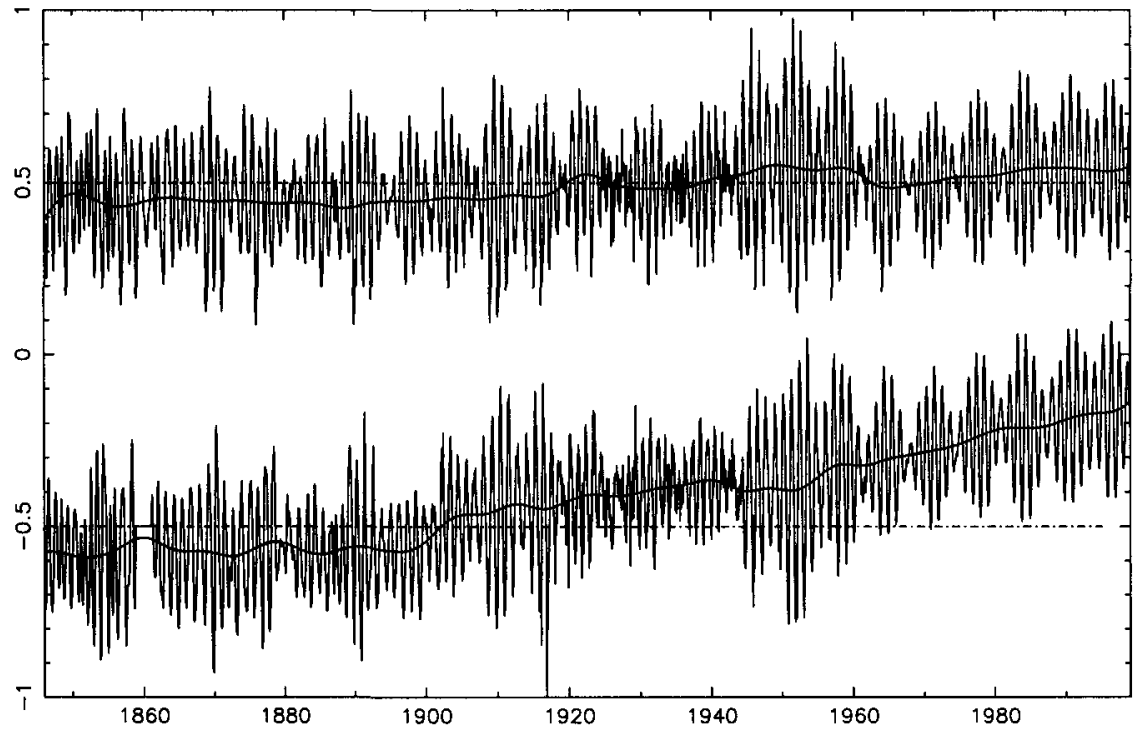

Figure 1. EOP(IERS) C01: X and Y pole Components, UNIT $=1^{\prime \prime}$ of degree.

dominated by both a forced seasonal term and the free Chandler motion. The beating of these two periods yields a 6-year oscillation visible on the figure.

A least-squares fit analysis using a gradient search was used (Bevington, $1969)$. It shows that the mean period is 435 days $( \pm 12 d)$ for $x$ and $y$ pole components of the Chandler term while it is 365 days $( \pm 7 \mathrm{~d})$ for the annual term. As it was already remarked, the Chandler term is variable over the period 19201940 (Figure 2). The technique of Wavelet Transform Analysis was extensively used in geodynanics (Gambis, 1992). Such analysis, using Torrence and Compo's (1998) procedure was done for the X-pole component (Figure 3). It shows the temporal time-scale evolution of the frequency spectrum between 0.5 and 2.5 years.

\section{Combination of EOP series, permanent solutions}

The maintenance of the reference system's consistency and its long-term stability require long and homogeneous EOP series. The realization of combined solutions must take advantage of the availability and the qualities of the independent series at various time scales. For practical reasons also linked to statistical applications, these series are given at equidistant intervals ( 1 day). They are assumed to contain no jump and negligible systematic errors. At least 3 independant techniques are thus highly desirable for that purpose. The contribution of these 3 techniques to geodynamics is important for their complementarity but also for some aspects linked to redundancy in order to eliminate systematic effects. 


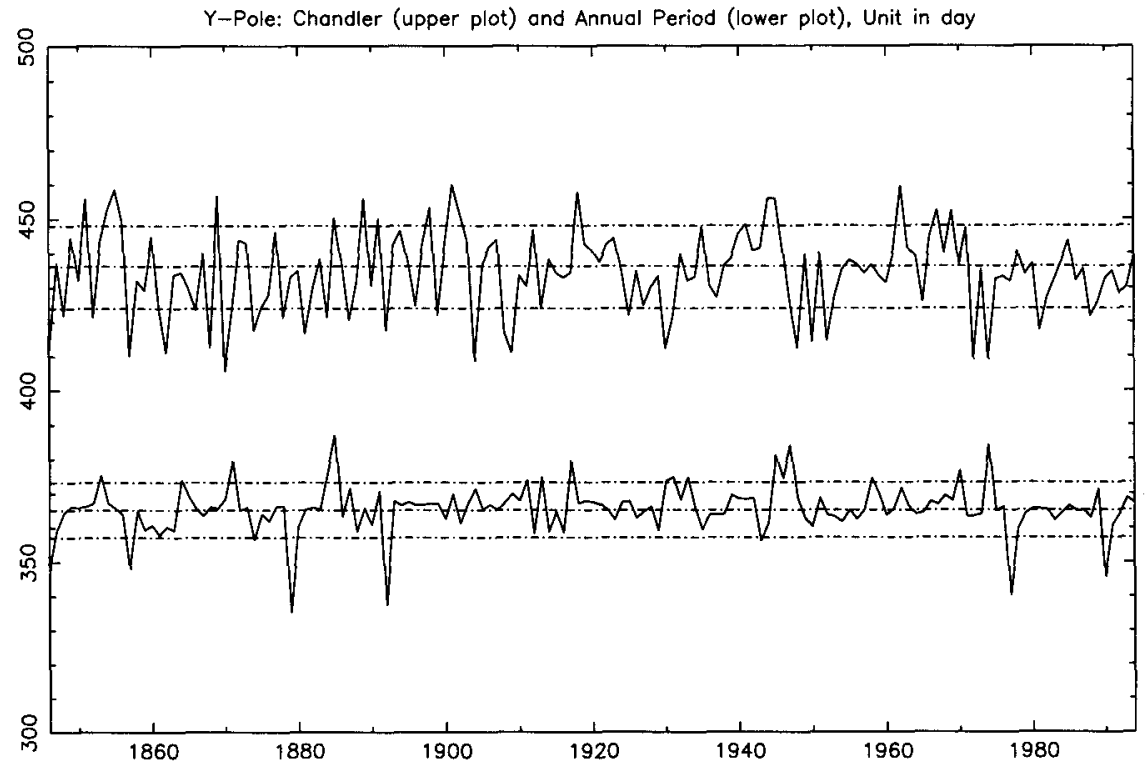

Figure 2. Evolution since 1846 of respectively the Chandler and Seasonal periods for $\mathrm{X}$ and $\mathrm{Y}$ component.

a. EOP(IERS) CO1, X-Pole component

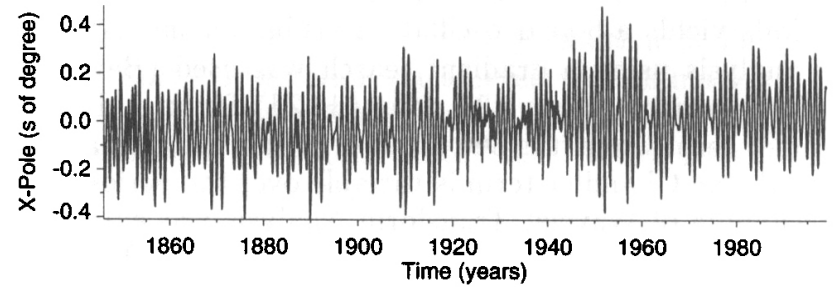

b. Wavelet Power Spectrum

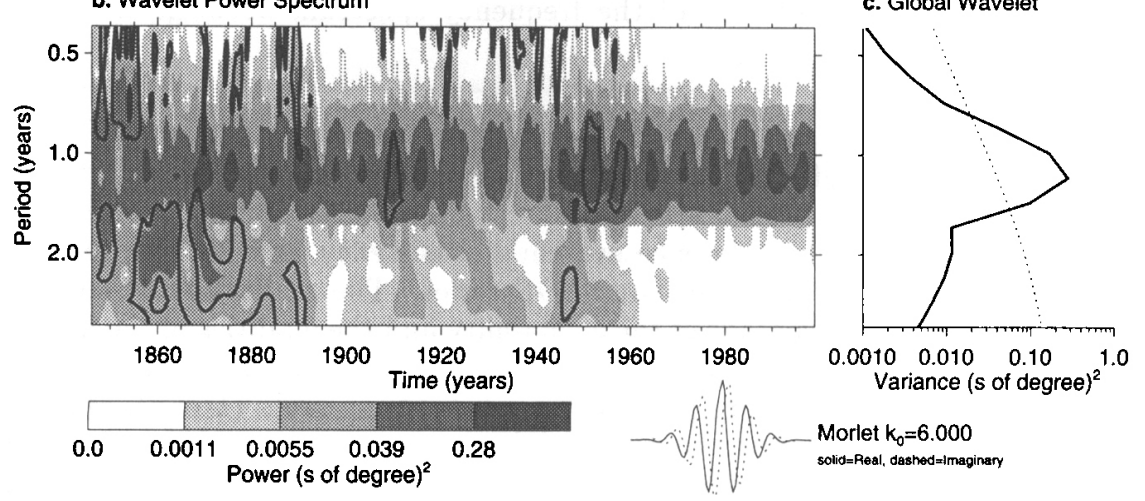

Figure 3. Wavelet Transform of X-Pole component of EOP(IERS) C01 (Torrence and Compo, 1998). 
The first step in the general procedure for deriving the IERS/CB multitechnique combined solution is the evaluation for each solution of the correction of systematic errors, bias and drift in order to translate it into the IERS system. A known source of relative drifts in $\mathrm{x}, \mathrm{y}$ and UT1-UTC is the variety of processes chosen by the analysis centres to control the time evolution of the adjusted terrestrial reference frames, complicated by the sampling of the tectonic plates and plate margins by the actual observing networks. The formal uncertainties estimated by the analysis centers being an internal consistency value, an external calibration has usually to be made in order to reflect the real uncertainty of the estimates. This is done using the Allan variance analysis (Gray and Allan, 1974) of the differences between series without any reference to a combined series. When three or more series of similar quality and time resolution can be differenced, the pair variance of the noise of each series can be evaluated, provided that their errors can be considered to be statistically independent. The pair variance thus obtained is used as an estimate of the uncertainty of a single determination in a given series. Its ratio with the rms formal uncertainty over the same period provides a scaling factor, on which the weighting of the combined individual results is based. Consequently a scaling factor is given to the series. Weights of the series entering the combined solution are thus estimated. The 3 main techniques (VLBI, SLR and GPS) have about the same contribution to the polar motion series whereas for UT and celestial pole offsets the unique contributor is VLBI. Table 2 represents the RMS agreement of these series with the $\mathrm{C} 04$ combined solution for both pole components. Note that these estimates are all smaller than 0.20 mas.

Table 2. RMS agreement with the combined IERS/CB solution.

\begin{tabular}{lcc}
\hline \hline Series & x pole (mas) & y pole (mas) \\
\hline GPS (IERS) & 0.17 & 0.12 \\
VLBI (USNO) & 0.17 & 0.13 \\
VLBI (IAA) & 0.18 & 0.16 \\
SLR (CSR) & 0.18 & 0.18 \\
\hline
\end{tabular}

Other series, based on normal points solutions given at various time intervals, are also proposed to users, i.e. C02, C03 (Gambis, 1996a and 1996b; Eisop and Gambis, 1997). These series are consistent. They use the full correlation matrix when available. Recently there were new developments in the normal point series $\mathrm{C} 02$ and $\mathrm{C} 03$. In these series, the estimation of the solution, given for the central dates of the n-day interval is made using a least-square fit for all EOP components. Although this estimation has been extensively used for data analysis, it has some drawbacks linked to problems of ill-conditioning and in the non-robust detection of outliers. Alternative methods based on robust estimators like M-Huber can be used (Bougeard M.L et al., 1999). These estimators are a generalization of both the L1 and L2 class. They have been implemented in our analyses and are now currently used (Bougeard M.L et al., 1999). 


\section{Predictions}

Different approaches are used at the Central Bureau for predicting the Earth rotation parameters.

Polar Motion: the formalism uses at first a floating period fit for both the Chandler and annual components estimation over a past time interval of several years. An autoregressive filter is then applied on the short-term residuals series and used for the prediction. The predictions of the nutation offsets $\mathrm{dPsi}$ and dEps are based on an empirical model (Conventions 1996).

Universal Time: the present formalism used is based on the assumption that the long-term fluctuations (annual and semiannual) of the preceding year are valid over the next few months. For short-term variations prediction, an autoregressive process is used. Table 3 shows the current accuracy of the EOP solutions.

Table 3. Assessment of the predictions precision over 1998.

\begin{tabular}{cccc}
\hline \hline & Pole(mas) & UT (.1 ms) & Celest. Pole Offsets(mas) \\
\hline $10 \mathrm{~d}$ & 4.0 & 20.0 & 0.3 \\
\hline $30 \mathrm{~d}$ & 9.0 & 40.0 & 0.3 \\
\hline
\end{tabular}

The application of an alternative procedure based on non-linear analysis (Frede, 1999) is under investigation (Frede, 1999). It relies on the chaotic nature of the Earth's rotational dynamical regime over a couple of weeks. Nonlinear time series analysis techniques have been applied to daily observations of the length of day (LOD) and polar motion components spanning more than 27 years and filtered in order to keep the period range (approximatively 100 days). It was shown that some theoretical prediction horizons cannot be passed beyond by any tentative forecast of the the EOP evolution. Horizons of 11 days for LOD and about 8 to 9 days for polar motion are found beyond which prediction errors will be of the order of the RMS of the filtered EOP series, i.e. respectively $0.12 \mathrm{~ms}$ and 2 mas. The stability of the reconstructed orbits is analyzed by determining their local Lyapunov exponents. It was shown that the Earth's rotational state experiences large changes in stability with regard to the average chaotic regime. Moreover the local prediction horizons, as deduced from the local Lyapunov exponents, occasionally drop down to about 3.3 days for LOD in the years 1982-1984, 2.6 days for polar motion. The main transitory stability perturbations of the Earth rotation are clearly related to El Niño and La Niña events (e.g. 1982-1984, 1972-1973). Preliminary developments are being carried out for application to operationnal predictions.

\section{Conclusion}

Long-term Earth orientation parameters series are derived from the combination and integration of all available data in a consistent way. A continuous composite series of polar motion components extending from 1846 until now EOP (IERS) 
C01 is available at the Earth Orientation Section of the Central Bureau of the IERS. This series is the basis of the IERS system.

Independent techniques are highly desirable to monitor the Earth rotation for their complementarity aspects but also to allow maintainance of a reliable link between the primary reference frames, terrestrial and celestial, and to separate true geophysical signals from systematic fluctuations.

GPS is now the technique of choice to monitor polar motion and highfrequency variations of UT1. For long-term variations of UT1 and nutation, VLBI techniques are still essential. Homogeneous polar motion SLR series, available since the launch of Lageos in 1976 also bring a significant contribution to the long-term stability of the IERS system.

The evolution in the respective contribution of the observing techniques and the increasing temporal resolution and accuracies obtained by the techniques make it necessary to maintain and constantly improve the combination procedures. New developments based on the use of the M-Huber norm are being applied. They are an alternative to the L2 norm usually used. Mixing the temporal series in an optimal way is a permanent challenge.

\section{References}

Bevington P.R., 1969, Data reduction and error analysis for the physical sciences, McGraw-Hill Book Company, New York, USA.

Bougeard M.L, D. Gambis and R. Ray, 1999, Algorithms for box constrained Mestimation: fitting large data sets with applications to Earth Orientation Parameters series, subm. to Physics and Chemistry of the Earth.

Eisop E. And D. Gambis, 1997: The combined solutions of the IERS Central Bureau, Proc. Journées Systèmes de Référence, Praha, p. 104.

Fedorov, E.P., Korsun, A.A, Mayor, S.P., Pantschenko, N.I., Tarady, V.K., and Yatskiv,Y.S., 1972: Dvizhenie polyusa Zemli s 1890.0 po 1969.0 . Naukova

Frede V., 1999, PhD thesis, Paris Observatory.

Gambis D., 1992, Wavelet transform analysis of the length of the day and the El Niño/Southern oscillation variations at intraseasonal and interannual time scales, Ann. geophys., 10, 429-437.

Gambis D., 1996: Multi-technique EOP combinations, Proceedings of the 1996 IGS Analysis Center Workshop, Silver Spring. MD, edited by P. Van Scoy and R.E. Neilan, Pasadena, CA, JPL, JPL Publication 96-23.

Gambis D., 1996, Monitoring Earth Rotation using various techniques, current results and future prospects, Proc. Coll. IAU 165, Dynamics and astrometry of natural and artificial celestial bodies.

Gray, J.E. and Allan, D.W. 1974: A method for estimating the frequency stability of an individual oscillator. Proc 8th Ann. Symp. on Frequency Control, 2439, 277-287. Dumka, Kiev. [English translation of the text available].

IERS Conventions, 1996, McCarthy (ed.).

Torrence, C. and G.P. Compo, 1998: A Practical Guide to Wavelet Analysis. Bull. Amer. Meteor. Soc., 79,61-78. 
Vondrák J., Ron C., Pesek I., Cepek A., 1995, Astron. and Astrophys., 297, 899-906. 